\title{
A 16th Century Escherichia Coli Draft Genome Associated with an Opportunistic Bile Infection
}

George Long ( $\sim$ longg2@mcmaster.ca )

McMaster University https://orcid.org/0000-0002-9088-9848

Jeniffer Klunk

Ana Duggan

Madeline Tapson

Valentina Giuffra

Lavinia Gazzè

Antonio Fornaciari

Sebastian Duchene

University of Melbourne https://orcid.org/0000-0002-2863-0907

Gino Fornaciari

Olivier Clermont

Paris Diderot University

Erick Denamur

Université de Paris

Brian Golding

McMaster University

Hendrik Poinar

McMaster University https://orcid.org/0000-0002-0314-4160

Article

Keywords:

Posted Date: March 7th, 2022

DOI: https://doi.org/10.21203/rs.3.rs-1409634/v1

License: (9) This work is licensed under a Creative Commons Attribution 4.0 International License. Read Full License

Version of Record: A version of this preprint was published at Communications Biology on June 16th, 2022. See the published version at https://doi.org/10.1038/s42003-022-03527-1. 


\title{
A $16^{\text {th }}$ Century Escherichia coli draft genome associated with an opportunistic bile infection
}

\author{
George S. Long ${ }^{1,2,{ }^{*}, \dagger}$, Jennifer Klunk ${ }^{1,2,3,}{ }^{*}$, Ana T. Duggan ${ }^{2,4}$, Madeline Tapson ${ }^{2,3}$, \\ Valentina Giuffra ${ }^{5}$, Lavinia Gazzè ${ }^{6}$, Antonio Fornaciari ${ }^{7}$, Sebastian Duchene $^{8}$, \\ Gino Fornaciari ${ }^{7}$, Olivier Clermont ${ }^{9}$, Erick Denamur ${ }^{9,10, \dagger}$, \\ G. Brian Golding ${ }^{1}$, Hendrik Poinar $2,11,12, \dagger$
}

March 1, 2022

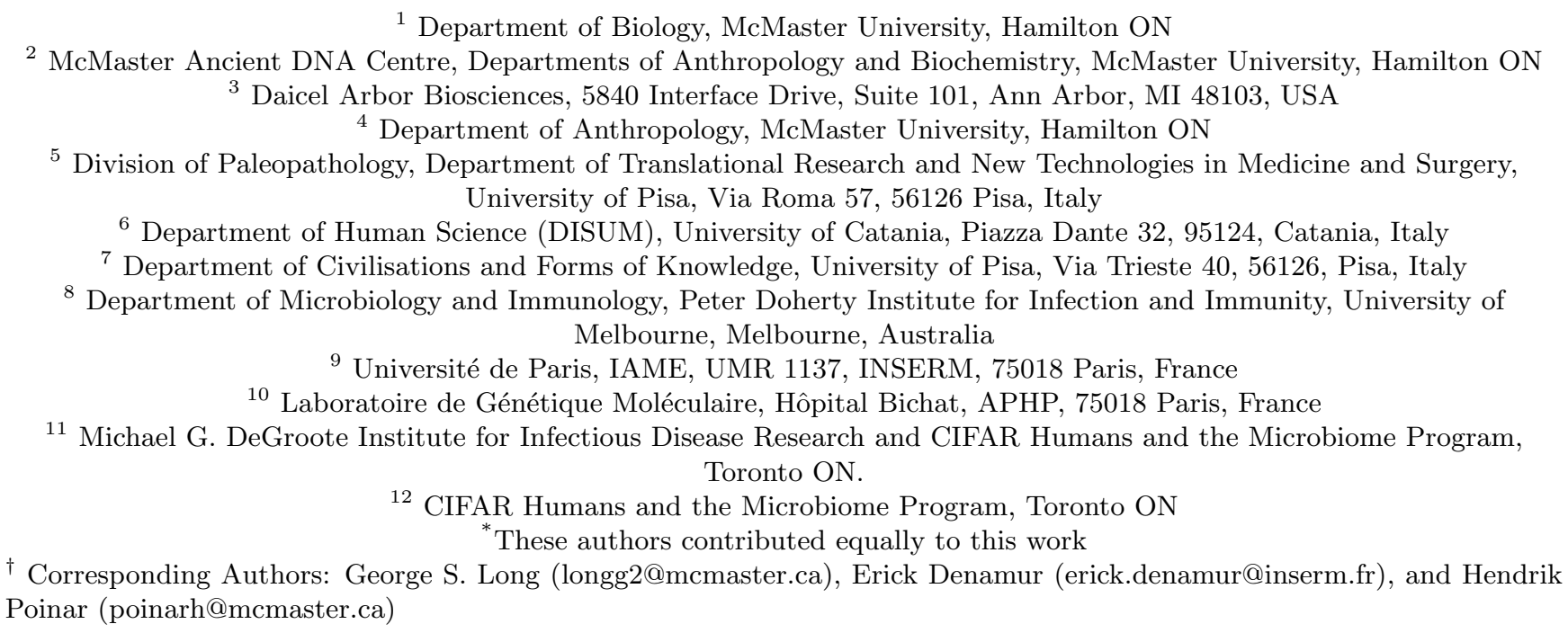

\begin{abstract}
Escherichia coli - one of the most characterized bacteria and a major public health concern - remains invisible across the temporal landscape. Here, we present the meticulous reconstruction of the first ancient E. coli genome from a $16^{\text {th }}$ century gallstone from an Italian mummy with chronic cholecystitis. We isolated ancient DNA and reconstructed the ancient E. coli genome. It consisted of one chromosome of 4446 genes and two putative plasmids with 52 genes. The E. coli strain belonged to the phylogroup A and an exceptionally rare sequence type 4995. The Type VI secretion system component genes appears to be horizontally acquired from Klebsiella aerogenes, however we could not identify any pathovar specific genes were detected nor any antibiotic resistances. A sepsis mouse assay performed using a closely related contemporary E. coli strain was avirulent. Our reconstruction of this ancient E. coli helps paint a more complete picture of the burden of opportunistic infections of the past.
\end{abstract}

\section{Introduction}

The recovery of ancient pathogen DNA (aDNA) from human victims has almost exclusively focused on historically significant mortality events, such as the Black Death, revealing the evolutionary history of canonical pathogens such as Yersinia pestis, Mycobacterium tuberculosis ${ }^{1}$, and Variola virus. In contrast, much human morbidity and mortality is the result of opportunistic infections, ones that often remain invisible in the past. Opportunistic pathogens, those without historical record - such as Escherichia coli, Pseudomonas aeruginosa, and Staphylococcus aureus - have been understudied relative to their contemporary burdens on people today 2 . They are defined by their ability to infect during periods of stress, imbalance, or disturbance while being otherwise commensal or saprophytic ${ }^{3}$. Opportunistic infections likely played an important role on human mortality in our shared past and thus have had a broader impact on human health than can be or has been measured. 
A confounding factor in the identification of historically understudied pathogens is that the resultant infections were likely primarily opportunistic. That is, they colonized their host environments asymptomatically, leaving no identifiable pathologies and as such are not easily identified in human remains ${ }^{4}$. Ancient DNA studies typically focus on obligatory pathogens such as M. leprae, Salmonella enterica, and Y.pestis that are easily correlated with pathologically distinct, or historically relevant mortality events and as such are easily distinguished as foreign in ancient human metagenomic DNA read data ${ }^{547}$. Opportunistic infections have the added burden of authentication due to their ubiquity as environmental contaminants and of modern commensal strains. Historical identification of these pathogens would allow for a careful assessment of their evolutionary history and the commensal-pathogen continuum defined by gene content gain and loss as strains modulate toward or away from host virulence.

E. coli is one such pathogen. It is a common commensal bacteria found in vertebrate gut microbiome $\mathrm{e}^{8}$ that can also become an opportunistic pathogen under specific conditions ${ }^{9}$. E. coli has such a large impact on our health care systems that it is the subject of several vaccine development efforts to mitigate the effects of the most pathogenic strains 10 . Having been responsible for several food poisoning outbreaks and becoming a leading pathogen for deaths caused by antimicrobial resistance, E. coli is thus a key bacterium used in public health surveillance 11 .

A global sampling of E. coli strains produces a tree with several unique phylogenetic groups and interspersed pathovars ${ }^{12}$. While the phylogenetic relationships between strains are constructed based on genetic similarity, pathovars are defined by the virulence traits of their members. In many cases, members of the same pathovar do cluster together in the same clade, however, as virulence genes can be acquired horizontally, some pathovars - like enteroaggregative E. coli - are distributed across multiple phylogenetic groups ${ }^{12}$. This striking diversity and the evolutionary transitory states among E. coli strains highlight their genomic plasticity and evolutionary versatility along this aforementioned continuum. Ancient E. coli genomes would provide useful insights into the forces that influence the emergence of commensalism and pathogenicity in bacteria. Here, we describe the reconstruction of a $16^{\text {th }}$ century E. coli genome characterized from the gallstone of an Italian noble - Giovani d'Avalos (1538 - 1586) highlighting a genome with commensal characteristics.

\section{Results}

\section{The Mummified Remains}

In 1983, the mummified remains of several Italian nobles were recovered from the Abbey of Saint Domenico Maggiore in Naples, Italy. A careful paleopathological and histological survey of one of the individuals - Giovani d'Avalos (NASD1), a Neapolitan noble who died in 1586 at the age of 48 (Supplemental figure 1) - revealed thickened gallbladder walls, Rokitanski-Aschoff sinuses, and several intact gallstones ${ }^{13}$. These features suggested that NASD1 may have suffered from chronic cholecystitis ${ }^{14}$. While not the only cause of cholecystitis, chronic bacterial infections from E. coli, Bacteroides spp., and Clostridium spp. can lead to the formation of gallstones ${ }^{15}$. These infections are typically indicated by a brown pigment, as can be seen in the gallstone from NASD1 ${ }^{[15}$ (figure 1 A-B).

\section{Authenticating the Ancient DNA}

Metagenomic profiles generated from the DNA extracts using Kraken 2 provided preliminary evidence for the substantial and increasing presence of Enterobacteriales in digest rounds 2 (outer layer), 3-4 (middle layer), and 5-6 (inner layer) from the gallstone while indicating its absence in the tissue samples of NASD1 (Supplemental figure 2). Enterobacteriales reads are also present in digest round 1 however they are comparable with those in the reagent blanks (Supplemental figure 2), thus likely to be a mixture of endogenous and contaminant DNA such as Bradyrhizobium. Species-level indication suggested that E. coli made up the largest proportion of reads identified as Enterobacteriales. Fortunately, E. coli specific reads are virtually absent $(\leq 0.002 \%)$ from the blanks and present in the second through sixth digests. In comparison, other taxa such as Alcaligenes and Bradyrhizobium were present in both the experimental samples and the blanks and are likely to be contaminants. Human assigned reads were present in all digests, but were also detected in the blanks. Klebsiella aerogenes was also detected, however only in digest rounds 3-4. To assess the authenticity of the Human, E. coli, and K. aerogenes reads we examined the deamination and depurination kinetics (Figure 1 C-D, Supplemental Figure 3).

\section{Reconstructing the Ancient E. coli Genome and Putative Plasmids}

The reconstructed ancient E. coli genome had a mean read depth of $28.46 \pm 5.28 \times$, mean heterozygosity of $7.66 *$ $10^{-4} \pm 3.39 * 10^{-3}, 4446$ genes when mapped to the E. coli pan-genome dataset and a total estimated size of 
Table 1: Read coverage metrics for the $E$. coli pan-genome, FSIS11816402, K-12 MG1655, CP019906.1, and CP012732.1 references.

\begin{tabular}{l|cc}
\hline Sequence & \% Positions with Coverage $\geq 1 \times$ & Mean Coverage \\
\hline Pan-Genome & $97.93 \%$ & $28.46 \pm 5.28 \times$ \\
FSIS11816402 & $77.84 \%$ & $18.00 \pm 8.20 \times$ \\
K-12 MG1655 & $78.30 \%$ & $20.94 \pm 13.89 \times$ \\
CP019906.1 & $80.62 \%$ & $20.95 \pm 13.17 \times$ \\
CP012732.1 & $64.01 \%$ & $16.46 \pm 14.58 \times$ \\
\hline
\end{tabular}

FSIS11816402 used the mean of means greater than $1 \times$ as it was an assembly.

4, 050, 429bp when the detected genes are concatenated. Significant differences $(P<0.001)$ were detected when comparing read depths of the core (3122 genes, $28.73 \pm 3.47 \times)$ and accessory $(1324$ genes, $27.82 \pm 8.04 \times)$ genomes (figure 2 A, table 1); the same was true for their mean heterozygosity with the core at $2.74 * 10^{-4} \pm 1.16 * 10^{-3}$ and accessory genome with $1.95 * 10^{-3} \pm 5.79 * 10^{-3}$ (Supplemental Figure 4). Eighty-three genes were identified has having a substantially (at least two standard deviations) deeper gene coverage. These results were then compared to the closely related A phylogroup E. coli genomes of FSIS11816402, an sequence type (ST) 4995 strain and K-12 MG1655, an ST10 strain (figure 2B, Supplemental figure 5). Both genome comparisons returned a lower mean depth with a larger variance of coverage. This is especially true for FSIS11816402 as $25.24 \%$ of its contigs had a mean coverage less than $1 \times$ (Supplemental figure 6).

We identified two potential plasmids within the assembled scaffolds (see appendix for details) with MOB-suite: E. coli MDR_56 plasmid unnamed 5 (CP019906.1) and S. flexneri 1a strain 0228 plasmid (CP012732.1). Subsequent mappings of the E. coli reads back to these reference-plasmids confirmed their presence in the ancient strain and authenticated their origin (figure 2 C,Supplemental figure 5, Supplemental figure 7). One plasmid, CP019906.1 had a read coverage similar to that of K-12 $(20.95 \pm 13.17 \times)$ and 34 genes, whereas, CP012732.1 had lower coverage $(16.46 \pm 14.58 \times)$ and only 19 genes. These plasmids contain regions with no read coverage - including a $\sim 6.6 \mathrm{Kbp}$ region in CP012732.1 - but we could not confirm if the genes were part of the chromosome.

\section{Identification of Co-Circulating K. aerogenes DNA}

A subset of the sequenced reads were also classified as belonging to K. aerogenes by Kraken2. To confirm or refute this result, unmapped reads were aligned against the K. aerogenes (NC_015663.1) reference genome. The overall read depth $(0.38 \pm 2.89 \times)$ and genome coverage was low $(3.43 \%)$, suggesting that only a subset of genes from K. aeorgenes was present. However, a $38 \mathrm{Kbp}$ section of the genome (figure 2D) had a read mapping depth similar to the ancient $E$. coli results $(20.36 \pm 13.56 \times)$.

\section{Phylogenetic Reconstruction}

In order to place the ancient E. coli within the global phylogeny and help identify the strain we produced a core SNP alignment of $451 \mathrm{E}$. coli and four Shigella genomes representing the current breadth of E. coli diversity. After removing redundancy, the resulting phylogeny contained 107 genomes. This pruned sample set was then used to create a new alignment of 5007 core SNPs which returned an overall topology resembling previously published results and placed the ancient strain into a strongly supported phylogeny within phylogroup A (figure $3 \mathrm{~A}$ ).

To more carefully refine the position of the ancient strain within group A, we generated a reduced ML tree using 94 genomes (figure 3B) consisting of 291 SNPs. The ancient genome clustered closely with the ST4995 strains with some statistical support (bootstrap support of 65\%), signifying that it is likely part of the same sequence type (table 2). An even further refined ML tree was then generated using the 22 available ST4995 genomes from Enterobase (figure $3 \mathrm{C}$ ). The core SNP alignment contained 16, 026 nucleotides, providing a much greater resolution than the global and A0 subgroup alignments. The ancient strain clearly clusters within the ST4995 genomes.

We scanned for temporal signal across all phylogenies using TEMPest and found one when the phylogeny was restricted to the ST4995 genomes (Supplemental figure 8), a result confirmed a date randomization test in LSD $(P=0.003)$. The ST4995 phylogeny (figure $3 \mathrm{C})$ suggests an $11^{\text {th }}$ century $(1027[787,1220] \mathrm{CE})$ tMCRA for ST4995 strains, nearly 500 years before the diversification of the modern strains. The estimated evolutionary rate across the phylogeny was $2.555 \times 10^{-6}\left[1.567 \times 10^{-6}, 3.992 \times 10^{-6}\right]$ subs/site/year, similar to previously published results $\frac{16}{\text {. }}$. 
Table 2: SNP distances of seven strains from the ST4995 phylogeny.

\begin{tabular}{|c|c|c|c|c|c|c|c|}
\hline Genomes & CFSAN051544 & Ancient Ecoli & FSIS11816402 & ATCC 11229 & ESC_SA9272AA & ESC_DB2295AA & ESC_CA2237AA \\
\hline CFSAN051544 & 0 & 771 & 869 & 920 & 971 & 1012 & 1024 \\
\hline Ancient Ecoli & 771 & 0 & 419 & 503 & 554 & 596 & 607 \\
\hline FSIS11816402 & 869 & 419 & 0 & 588 & 639 & 681 & 692 \\
\hline ATCC 11229 & 920 & 503 & 588 & 0 & 215 & 219 & 230 \\
\hline ESC_SA9272AA & 971 & 554 & 639 & 215 & 0 & 308 & 319 \\
\hline ESC_DB2295AA & 1012 & 596 & 681 & 219 & 308 & 0 & 323 \\
\hline ESC_CA2237AA & 1024 & 607 & 692 & 230 & 319 & 323 & 0 \\
\hline
\end{tabular}

CFSAN051544 was the outgroup of the phylogeny.

Multilocus sequence and typing of our ancient E. coli genome confirmed its placement in the ST4995 - a rare sequence type with only 22 strains in the Enterobase database which encompasses 182, 476 entries $\frac{17}{17}$. It exhbited a Onovel15:H? serotype, similar to its sister taxa (see appendix for full results). A different fim $H$ variant - fimH86 - was present in our E. coli strain.

\section{Global Gene Content}

The majority (95\%) of the E. coli strains contained 3190 core genes, 3122 of which were detected in our ancient genome (Supplemental figure 9). An enrichment analysis of the missing core genes revealed a significant lack of flagellar assembly genes $(P=0.0445)$, however, less protein-protein interactions were detected in the network than expected $(P>0.05)$. An analysis of regions with mapped ancient reads in CP019906.1 revealed the presence of genes involved in biofilm formation (NP_311382.1) and a multidrug efflux pump (acrD). For CP012732.1, in contrast, reads mapped to pseudogenes likely related to osmoprotection and transcription regulations.

A presence/absence $(\mathrm{P} / \mathrm{A})$ analysis of the accessory genome recapitulates the results from the ML phylogeny results (figure $4 \mathrm{~A}$ ) and previously published results $\frac{18}{1}$. The accessory PCoA indicates that the genome is a member of phylogroup A and an ancestral member of ST4995. The latter is confirmed by a P/A analysis of a ST4995-only pan-genome (Supplemental figure 10).

\section{Virulence Results}

A total of 91 virulence factors were identified in the ancient genome, 37 of which were not found in E. coli K-12 MG1655 (see Table 3 for gene families with more than one hit). Type VI secretion system (T6SS) components consisted as the majority of these genes and contained high copy numbers. The T6SS - formed by the tss gene family as well as $h c p, \operatorname{vasK}, \operatorname{vgr} G$ - mediates antagonistic interactions between competing bacteria. In addition to its role in bacterial killing, T6SS is involved in interbacterial signaling, biofilm formation, and phage defense $\mathrm{e}^{19}$. Parts of a type III secretion system were also detected at much lower levels.

Table 3: Identified virulence factors which contained genes not found in K-12 MG1655

\begin{tabular}{cccll}
\hline Gene Family & Genes & Mean Coverage & Function & Pathovar \\
\hline tss & 13 & $38.21 \pm 10.84 \times$ & Type VI Secretion System ${ }^{2}$ & EAEC \\
elf & 4 & $31.08 \pm 2.87 \times$ & Fimbriae & EAEC, EHEC, ExPEC \\
hcp & 3 & $36.02 \pm 11.95 \times$ & Type VI Secretion System & EAEC, EHEC, ExPEC \\
cfa & 2 & $23.01 \pm 12.99 \times$ & Fimbriae & EAEC, EHEC, ExPEC \\
vas & 2 & $51.62 \pm 1.31 \times$ & Type VI Secretion System & EAEC \\
vgr & 2 & $47.53 \pm 1.00 \times$ & Type VI Secretion System & EAEC \\
\hline
\end{tabular}

Only families which had more than one distinct gene are present. EAEC: Enteroaggregative E. coli; EHEC: Enterohemorrhagic E. coli; ExPEC: Extraintestinal Pathogenic E. coli.

We also observed incomplete virulence gene complements involved in several mechanisms. Several genes belonging to the ecp and fim fimbriae families were found in the ancient genome. While they are involved in virulence $\mathrm{e}^{9120121}$, they can also be present in commensal strains. Fimbriae known to be associated with virulence such as $c f a B$ and $c s g$ were also identified ${ }^{20}$ yet they do not provide enough information to pinpoint the specific pathovar of the ancient

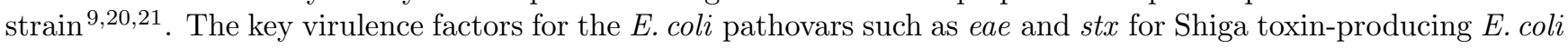


(STEC), heat labile and heat stable genes for Enterotoxigenic E. coli (ETEC), and the type III secretion system in EPEC and EHEC were absent ${ }^{9}$.

In contrast ast $A$, a key virulence factor in Enteroaggregativce E. coli (EAEC) ${ }^{22}$, was detected in the ancient genome. In combination with evidence that T6SS is commonly found in EAEC strains ${ }^{9}$, this may indicate that the ancient strain was been a member of this pathovar. The remaining EAEC virulence factors in the pan-genome $a g g A, a g g R$, and $a a p$ - however were not detected 22 . The ancient genome also contains a complete enterobactin system which is responsible for iron uptake ${ }^{20}$. The enterobactin system and ast $A$ gene were also found in $E$. coli K-12 MG1655, once again indicating that they do not confer enough virulence on their own.

\section{Mouse Sepsis Model}

To evaluate the extra-intestinal virulence potential of ST4995 we tested a close relative (507 core SNPs separating the two strains) of the ancient genome, the reference strain ATCC11229 (see figure 3B, Supplemental figure 11 for similarities to the modern strain) in a well-calibrated mouse sepsis assay where 10 mice were inoculated with the strain and their death recorded 23 . None of the mice were killed by ATCC11229, whereas all of the mice infected by the positive control B2 phylogroup CFT073 strain were killed. The phenotype of the strain which killed the mice is linked to the presence of specific extra-intestinal virulence genes with a major role of iron capture system encoding genes as those bared by the high-pathogenicity island (HPI) ${ }^{24125}$. This result is in agreement with the absence of typical extra-intestinal virulence genes present in the ATCC11229 and ancient strain.

\section{AMR profile of our Ancient E. coli}

We searched our ancient genome for the presence of antimicrobial resistance (AMR) genes using the Resistance Gene Identifier and found 47 distinct genes in the scaffold assembly of our ancient E. coli genome. Of these, 35 genes were also identified in the global pan-genome P/A analysis. Eight genes contained duplicates with $m d t B$ being the most commonly detected. The remaining subset spanned thirteen antimicrobial drug resistance classes with nine classes represented more than once (table 4). Resistance cassettes targeting the five most common drug classes were detected in the ancient E. coli strain. The majority of these genes are multi-drug efflux pumps, which is typical of E. coli $\underline{26}$. There were no unexpected AMR genes present in our ancient genome with all 35 being found in E. coli K-12 MG1655.

Table 4: AMR profile of the ancient E. coli.

\begin{tabular}{lrr}
\hline Resistances & With Efflux & No Efflux \\
\hline fluoroquinolone antibiotic & 17 & 1 \\
penam & 13 & 1 \\
tetracycline antibiotic & 10 & 1 \\
cephalosporin & 6 & 1 \\
peptide antibiotic & 5 & 3 \\
phenicol antibiotic & 5 & 1 \\
cephamycin & 4 & 1 \\
glycylcycline & 4 & 1 \\
rifamycin antibiotic & 4 & 1 \\
triclosan & 4 & 1 \\
carbapenem & 2 & 1 \\
penem & 2 & 1 \\
monobactam & 1 & 1 \\
\hline
\end{tabular}

Antibiotic results from an RGI 27 search of the E. coli scaffolds. A total of 29 genes were identified, with genes appearing multiple times. Only drug classes which were detected at least once without the use of antibiotic effluxes. A single gene can be present in multiple drug classes. 


\section{Discussion}

\section{A Clear Signal of DNA Damage}

The DNA isolated from the stone showed clear evidence of DNA damage. Specifically, the deamination plots for the ancient reads mapping to the $E$. coli pan-genome reference contained characteristic aDNA profiles which indicated increased deamination rates at or near the terminal bases (figure $1 \mathrm{C}$ ). In addition depurination kinetics plots of the E. coli mapped reads from libraries with sufficient E. coli read depth (digests 2, 3-4, 5-6) showed that fragments from internal sections of the gallstone (better protected from hydrolysis) were on average longer (34bp median in digest 2 to 37bp median in digests 5-6) (figure 1D). We obtained similar results for the K. aerogenes data across digests two through six. Interestingly, the mapped human reads lack these signature features (Supplemental figure 3 ). More importantly, however, is that they confirm that the E. coli and K. aerogenes sequences are indeed ancient and were not the result of modern contaminantion.

Two potential explanations exist for the lack of deamination signal in the mapped human reads. The first is that the sample likely contains a mixture of both contaminating and to a lesser degree authentic ancient human DNA. This is almost certainly the case for the first digest, as it represents the outer layer of the gallstone, which would have been subjected to contamination from handling, storage, sampling, etc. However, as we moved to deeper layers of the gallstone, presumably better protected from contamination, the lack of endogenous DNA was surprising. This is especially striking with the edit distances for both human and E. coli reads from across the different digests (Supplemental figure 12). The more likely explanation is that there are simply not enough human DNA reads for a meaningful damage analysis. Gallstones are typically formed from a combination of cholesterol, bile salts, and phosphatidylcholine ${ }^{15}$. Furthermore, in the highly acidic environment of the gallbladder, only bacteria that are specialized in surviving these conditions can thrive and potentially form a stone $\mathrm{e}^{15}$. Since gallstones are not formed from direct human cellular components its interior is likely virtually devoid of human DNA, which is very different from the results we obtained from the DNA of ancient abscesses 28 .

\section{An Intact Genome in Multiple Parts}

The gene depth results of the ancient E. coli genome confirms the lack of modern contaminants as they are part of a unimodal normal distribution. The sole exception to these coverage stats are eighty-three genes with substantially larger mean depths (Supplemental File 2). These genes consist of primarily hypothetical proteins (24.10\%), intergrases and transposons $(19.28 \%)$, and the T6SS $(13.25 \%)$. They also contain a significantly greater mean heterozygosity $\left(1.23 * 10^{-2} \pm 1.41 * 10^{-2}\right)$ than the rest of the ancient genome. These high copy number genes, however, are mainly accessory genes $(96.34 \%)$ which suggests - in combination with the greater heterozygosity and presence of transposons - that they were located in mobile elements shared between multiple bacterial species.

The plasmids we detected in the scaffold assembly, CP019906.1 and CP012732.1, had lower gene depth coverages than that for our reconstructed chromosome (Table11). Most plasmids are typically present in greater copy numbers than chromosomes ${ }^{29}$, however this was not the case as the ancient chromosome had a significantly $(P \approx 0)$ deeper mean coverage than CP019906.1 and CP012732.1. One possibility is that these two plasmids are actually not present, but rather the genes to which the ancient reads mapped were actually located in the ancient chromosome. This is supported by the fact that the mean gene depths for the plasmids falls firmly within the coverage distribution of the chromosome (Supplemental Table 2). This could explain large regions of the plasmids that lack any mapped reads, as well as the lack of reads mapping to intergenic regions (see $2 \mathrm{C}$ and ??B).

The reads mapping to the K. aeorgenes genome could represent a similar situation to the plasmid data. Interestingly, the $38 \mathrm{Kbp}$ region in the K. aerogenes chromosome which had a read depth similar yet significantly $(P<0.0001)$ different from the plasmids and $E$ coli chromosome $(20.36 \pm 13.56 \times)$ contains the Type VI Secretion System (T6SS), which is ubiquitous across Gram-negative bacteria and plays an important role in antagonistic interactions ${ }^{19}$. Given that T6SS is widespread among bacteria, its presence is not an exclusive marker for K. aerogenes. However, competitive read mapping between E. coli and K. aerogenes does suggest that this T6SS does indeed belong to K. aerogenes rather than the homologous system in E. coli suggesting a recent horizontal transfer, which is supported by comparison of their relative GC $\operatorname{contents}^{30}$ (Supplemental figure 13).

\section{The Temporal Evolution of ST4995}

The structure of our global phylogeny resembles those previously published ${ }^{31}$ and places the ancient genome in phylogroup A with meaningful support (100\% bootstrap for clade of three strains). Interestingly, other historical E. coli strains have also been placed in this phylogroup ${ }^{32}$ and it has been shown that commensal strain phylogroups 
is primarily driven by socioeconomic factors such as industrialization and diet ${ }^{8}$. Strains that have been isolated from humans living in less industrialized and more rural communities typically harbour strains falling within phylogroup A. The late Medieval date of our sample, along with its phylogenetic placement helps confirm its authenticity. In addition, a study of E. coli strains isolated from bile infections showed that they belong mainly to the phylogenetic A group 33 .

We identified a temporal signal in the reduced phylogeny (Fig $3 \mathrm{C}$ ) which provides additional context for the evolutionary history of ST4995 strains. The majority of the sequence type can trace their lineage to a strain from approximately $1592[1479,1690] \mathrm{CE}$, however, the divergence of our ancient genome and two others - ESC_AA9618AA_AS and ESC_VA4573AA_AS - has a deeper tMRCA ca. $956[689,1172]$ CE. Given the disparity in ages between the two clades, it is likely that ST4995 is actually composed of at least two subgroups. Evidence supporting this can be seen in a MDS of the P/A analysis of all existing ST4995 genomes (Supplementary figure 9).

\section{An Ancient Opportunistic Pathogen Fully Susceptible to Antibiotics}

The $\mathrm{P} / \mathrm{A}$ analysis of the accessory genes recapitulates the results from the ML phylogenies (figure $4 \mathrm{~A}$ ). The accessory $\mathrm{PCoA}$ confirms that the ancient strain is a member of phylogenetic group A while a subsequent $\mathrm{P} / \mathrm{A}$ analysis with a ST4995-only pan-genome confirms that the genome is a member of ST4995 (Supplemental figure 9). Phylogenetic group A - the group from which ST4995 stems - is a well described clade and is the most commonly isolated type from human commensals ${ }^{8}$. E. coli strain diversity is driven primarily by socioeconomic factors as individuals living in modern industrialized countries are more likely to carry B2 strains while those in less industrialized and rural communities harbor mostly $\mathrm{A}^{8}$. Given the time-period NASD1 lived, our ancient E. coli is a member phylogenetic group A.

No acquired AMR genes were detected in the ancient genome confirming the age of the strain. Post removal of the multi-drug efflux pumps from our genome, no other AMR genes were found when compared to E. coli K-12 MG1655 26. The latter is extremely pertinent as a strong resistance to a particular drug class requires several mechanisms ${ }^{\sqrt{34}}$. Thus, it is likely that only mild resistances to these antimicrobial compounds were needed $26 / 35$.

The identified ancient genome likely exhibited commensal characteristics as it shares most of its virulence factors with E. coli K-12 MG1655. The ancient strain lacks the canonical virulence factors for STEC and ETEC while also missing the components of the type III secretion system used in EHEC. Components of EAEC - ast $A$ and the T6SS ${ }^{9}$ - were detected, yet other key virulence factors were missing and ast $A$ was also detected in K-12 MG1655. Results from the $\mathrm{P} / \mathrm{A}$ analysis of the accessory genes with pathovar labels (figure $4 \mathrm{C}$ ) and the virulence genes (figure4 $\mathrm{D}$ ) presents a similar picture. The ancient genome is positioned in a relatively general cluster containing a variety of different pathovars which include EHEC, ETEC, ExPEC, and STEC. The mouse sepsis model further reinforces that the ancient genome was an opportunistic pathogen as the proxy strain - ATCC 11229 - did not infect any mice. This information, in combination with the identified virulence genes and $\mathrm{P} / \mathrm{A}$ clustering of the ancient E. coli suggests that the ancient genome is an opportunistic pathogen that acquired a K. aerogenes T6SS gene cassette during expansion in the gallbladder, consistent with previous work showing Klebsiella sp often associated with E. coli in contemporary bile infections ${ }^{36}$.

\section{Material \& Methods}

\section{Sample, DNA extraction, Library preparation and Sequencing}

We performed DNA extractions on a single gallstone - that had been isolated from the mummified remains of Giovani d'Avalos (NASD1) - in the clean-room facilities of the McMaster Ancient DNA Centre (McMaster University, Hamilton, Ontario, Canada) using methodologies specific for ancient DNA. We hypothesized that the successive mineralized layers of the stone would contain increasingly better-preserved DNA and thus subjected the gallstone to six successive rounds of demineralization and digestion $\frac{37}{37}$. Subsequently, DNA was extracted from $250 \mu L$ of the supernatant stemming from each round, which was then purified and prepared into dual-indexed libraries, sizeselected to remove artifacts, and sequenced on the Illumina HiSeq 1500 platform with $90 \mathrm{bp}$ paired-end reads $\frac{38 / 39}{3}$. Three additional tissue types from the same individual (bladder, small intestine, and lung) were processed and sequenced as above for comparative purposes. Full details of the methods can be found in the appendix.

\section{Bioinformatics}

Adapters were identified through AdapterRemoval ${ }^{40}$ with trimming and merging performed by leeHom ${ }^{41}$ using its aDNA setting. Sequencing lanes were pooled when applicable and Kraken $2^{\underline{42}}$ determined the overall metagenomic 
composition of the samples. This was done to characterize the metagenome and identify taxa of interest. A standard Kraken2 database with some modifications was used - a kmer size of 25bp and no minimizer - to account for the smaller read lengths of aDNA fragments. Reads underwent string deduplication using prinseq ${ }^{43}$ prior to the metagenomic analysis.

Samples were then mapped against the human genome $G R C h 38^{44}$ with BWA ${ }^{45}$ using previously published parameters $\frac{46}{4}$. A minimum length of $30 \mathrm{bp}$ and mapping quality of 30 was required for a read to be successfully matched. Fragments did not successfully map were then compared with an E. coli pan-genome using the same settings. The pan-genome was created with E. coli genomes from PATRIC $\stackrel{47}{ }$ and four Shigella genomes from NCBI. Specifically, genomes which were found in humans, chickens, cows, dogs, mice, or pigs and were determined to have a "good" genome quality (as defined by PATRIC) were selected resulting in 451 strains. These sequences were annotated with Prokka ${ }^{48}$ using the proteins from E. coli K-12 MG1655 (NC_000913.3) obtained from NCBI as trusted sequences. The resulting annotations were compiled by Roary ${ }^{49}$ to create the pan-genome. Paralogs were not split and a minimum blastP identity of $90 \%$ were used as additional settings. Unsuccessfully mapped human reads were also compared with Klebsiella aerogenes (NC_015663.1) as it was substantially - proportional abundance $\geq 1 \%$ - present in one of the digests while also being excluded from the blanks.

Successfully mapped human, E. coli, and K. aerogenes reads were deduplicated based on their $5^{\prime}$ and $3^{\prime}$ coordinates with bam-rmdup (https://bitbucket.org/ustenzel/biohazard-tools/src/master/). The deduplicated samples were pooled together based on their digest of origin $(1,2,3-4$, or 5-6). MapDamage $2.0 \sqrt{50}$ was used to estimate amount of deamination across the mapped reads. Fragment length distributions (FLD) and mapping mismatches were also extracted. Heterozygosity was tested by calling Single Nucleotide Polymorphisms (SNPs) using bcftools with ploidy set to two ${ }^{51}$ and a quality threshold of 100 .

Following authentication, an assembly was created with the complete set of mapped E. coli and K. aerogenes reads (i.e. not deduplicated) using SPAdes 3.14 .1 in isolate mode ${ }^{52}$ with customized kmer lengths - 9, 19, and 29. The resulting assembly was incomplete and highly fragmented scaffold. This was mostly due to the short median fragment lengths (35bp) of the input reads and the required kmer sizes. Nevertheless, the N50 of the assembly was 939bp with a total length of $3,917,339 \mathrm{bp}$ which is significantly shorter than the complete E. coli chromosomes in PATRIC $(5.00 \pm 0.517 \mathrm{Mbp})$. MOB-suite was used to identify potential plasmids in the assembly, however, the final identification was done by mapping to the reference plasmids 53 .

\section{Phylogenetic Analysis}

We created a SNP alignment using the E. coli genomes from PATRIC and the Shigella genomes using Snippy (https://github.com/tseemann/snippy) with E. coli EC42405 (CP043414.1) as the reference strain. Recombinant regions were removed and the resulting alignment used to create a maximum-likelihood (ML) phylogeny. The strains were labeled based on known E. coli phylogenetic groups. Upon determining the phylogroup, the subgroup A0 E. coli genomes were used to carefully position the ancient strain within the same clade. This subgroup is defined by the Clermont genotype ( + - - $)^{54}$. An additional seven enterotoxigenic E. coli strains obtained from von Mentzer et al. 2014 were also included in the phylogeny 5 . This phylogeny was created using the same process noted previously and was rooted using IAI1 B1 (NC_011741.1). The phylogeny of the ST4995 strains was created with the 22 ST4995 genomes available in Enterobase ${ }^{17}$ and rooted using the ST325 strain CFSAN051544 (SAMN05414627). To determine if the data contained any temporal signature, sampling dates or sequencing dates were assigned to the pruned phylogeny and a Root-to-tip (RTT) regression was performed for the global, A0 subgroup, and ST4995 phylogenies. The presence of temporal signal was then assessed via a date-randomization test. A molecular clock was fit to the data using a least-squares dating approach using LSD2 2657 .

\section{Gene Function Analysis}

To determine what genes were present in the ancient strain, we converted the read depths of the ancient E. coli pan-genome into a gene presence/absence $(\mathrm{P} / \mathrm{A})$ metric. We then used a conservative approach, identifying a gene as present if it had an average sequence depth of at least $10 \times$ with a coefficient of variation $(\mathrm{CV}) \leq 1$. A CV filter was used to remove genes which contained substantial regions of stacked reads. In comparison to a percent coverage threshold, a CV is more permissive with unmapped areas if the rest of the gene coverage is relatively consistent. An additional threshold of $\bar{x}+2 * s$ was used to identify genes with a high copy number. While still part of the ancient genome these genes could potentially belong to plasmids ${ }^{29}$ or represent gene amplification 58 .

The P/A matrix for modern strains was generated by Roary. A $95 \%$ core genome of the modern strains was identified and genes missing from our ancient strain were submitted to STRING ${ }^{59}$ to be functionally annotated using a network consisting of "high confidence" interactions. Virulence factors were identified using a curated set of 
known virulence genes ${ }^{60}$. Ambiguously named genes were identified (see appendix for methods) and we searched for the keywords "secretion, invasion, and enterotoxin". Genes with hits from this search were included with the virulence genes. To determine the pathovar of the ancient genome, relevant information was extracted from the PATRIC metadata.

We performed a $\mathrm{P} / \mathrm{A}$ analysis on the core, accessory genomes and the virulence genes. The strains were transformed into a binary distance matrix and clustered using a PCoA. To ensure that redundant genes were not included, two exclusion criteria were created: genes that were ubiquitously present were excluded, and genes not present in at least five genomes were removed from the accessory genome analysis. Of the $451 \mathrm{E}$. coli genomes, four were removed from the pan-genome analysis due to low gene counts (see Supplemental Figure 14 for gene counts and Supplemental File 1 for removed genomes). The Resistance Gene Identifier (RGI) $\stackrel{27}{ }$ with default settings identified potential antimicrobial resistances in the ancient strain. Only RGI entries returned due to gene homologies and present in the pan-genome were accepted. Both the identified virulence and resistance genes were compared to the archetypal E. coli strain K-12 MG1655. It is avirulent and susceptible to antibiotics 61 .

Ten 14-16 g (4-week-old) female mice OF1 from Charles River ${ }^{\circledR}$ (L'Arbresle, France) per strain received a subcutaneous injection of $0.2 \mathrm{ml}$ of bacterial suspension in the neck $\left(2 \times 10^{8}\right.$ colony forming unit). Time to death was recorded during the following 7 days. Mice surviving more than 7 days were considered cured and sacrificed. The E. coli CFT073 strain was used as a positive control killing all the inoculated mice whereas the E. coli K-12 MG1655 strain was used as a negative control for which all the inoculated mice survive ${ }^{\sqrt{23}}$. The protocol ( ${ }^{\circ}$ APAFIS\#4948) was approved by the French Ministry of Research and by the ethical committee for animal experiments. The ATCC11229 strain (AMC 198) was obtained from the Institut Pasteur collection (CIP 103795) and whole-genome sequenced using Illumina technology to verify the identity of the strain. This strain is of commensal origin and commonly used in bacterial resistance testing.

\section{Data Availability}

The raw sequencing data has been uploaded to NCBI as PRJNA810725 with supporting metadata located in Supplementary Table 1. The scripts and additional data used to analyze the data after pan-genome mapping are available at https://github.com/longg2/AncientEcoli.

\section{Acknowledgements}

We'd like to thank Wael Elhenawy for his correspondence and Sara Dion for her technical assistance in the mouse model.

\section{Funding}

Funding for this work was supported by an SSHRC Insight Grant, research funding from CIFAR's human and the microbiome program to HP and the Boris Family Fund. This work was also partially supported by the "Fondation pour la Recherche Médicale" Equipe FRM 2016, grant number DEQ20161136698 to ED.

\section{Competing interests}

The authors declare that they have no competing interests.

\section{Authors' contributions}

GSL and JK were responsible for the investigation, methodology, formal analysis, and original draft of this study. GSL also performed the data curation. MT, ATD, VG, LG, and AF aided in the investigation. ATD also contributed to the methodology. GF provided the sample. ED and OC validated the E. coli function and typing results. ED also provided funding for the mouse sepsis assay. SD validated the temporal signal and aided in its investigation. GBG supervised and provided computational resources. HP conceived and administered the project, provided funding, and also supervised. Everyone reviewed and edited the manuscript.

GSL and JK contributed equally to the study. 


\section{References}

1 Sebastián Duchêne, Simon YW Ho, Ann G Carmichael, Edward C Holmes, and Hendrik Poinar. The recovery, interpretation and use of ancient pathogen genomes. Current Biology, 30(19):R1215-R1231, 2020.

2 Michael D Parkins, Ranjani Somayaji, and Valerie J Waters. Epidemiology, biology, and impact of clonal Pseudomonas aeruginosa infections in cystic fibrosis. Clinical microbiology reviews, 31(4), 2018.

3 Yvonne Achermann, Ellie JC Goldstein, Tom Coenye, and Mark E Shirtliff. Propionibacterium acnes: from commensal to opportunistic biofilm-associated implant pathogen. Clinical microbiology reviews, 27(3):419-440, 2014.

4 James W Wood, George R Milner, Henry C Harpending, Kenneth M Weiss, Mark N Cohen, Leslie E Eisenberg, Dale L Hutchinson, Rimantas Jankauskas, Gintautas Cesnys, Gintautas Česnys, et al. The osteological paradox: problems of inferring prehistoric health from skeletal samples. Current anthropology, 33(4):343-370, 1992.

5 Ben Krause-Kyora, Marcel Nutsua, Lisa Boehme, Federica Pierini, Dorthe Dangvard Pedersen, Sabin-Christin Kornell, Dmitriy Drichel, Marion Bonazzi, Lena Möbus, Peter Tarp, et al. Ancient dna study reveals hla susceptibility locus for leprosy in medieval europeans. Nature communications, 9(1):1-11, 2018.

6 Xiyan Wu, Chao Ning, Felix M Key, Aida Andrades Valtueña, Aditya Kumar Lankapalli, Shizhu Gao, Xuan Yang, Fan Zhang, Linlin Liu, Zhongzhi Nie, et al. A 3,000-year-old, basal S. enterica lineage from bronze age xinjiang suggests spread along the proto-silk road. PLoS Pathogens, 17(9):e1009886, 2021.

7 David M Wagner, Jennifer Klunk, Michaela Harbeck, Alison Devault, Nicholas Waglechner, Jason W Sahl, Jacob Enk, Dawn N Birdsell, Melanie Kuch, Candice Lumibao, et al. Yersinia pestis and the plague of justinian 541-543 ad: a genomic analysis. The Lancet infectious diseases, 14(4):319-326, 2014.

8 Olivier Tenaillon, David Skurnik, Bertrand Picard, and Erick Denamur. The population genetics of commensal Escherichia coli. Nature Reviews Microbiology, 8(3):207-217, 2010.

9 Matthew A. Croxen and B. Brett Finlay. Molecular mechanisms of Escherichia coli pathogenicity. Nature Reviews Microbiology, 8(11):26-38, 2010. ISSN 1740-1534. doi: 10.1038/nrmicro2265.

10 Maricarmen Rojas-Lopez, Ricardo Monterio, Mariagrazia Pizza, Mickaël Desvaux, and Roberto Rosini. Intestinal pathogenic Escherichia coli: insights for vaccine development. Frontiers in microbiology, 9:440, 2018.

11 Christopher JL Murray, Kevin Shunji Ikuta, Fablina Sharara, Lucien Swetschinski, Gisela Robles Aguilar, Authia Gray, Chieh Han, Catherine Bisignano, Puja Rao, Eve Wool, et al. Global burden of bacterial antimicrobial resistance in 2019: a systematic analysis. The Lancet, 2022.

12 Erick Denamur, Olivier Clermont, Stéphane Bonacorsi, and David Gordon. The population genetics of pathogenic escherichia coli. Nature Reviews Microbiology, 19(1):37-54, 2021.

13 Gino Fornaciari, Luca Pollina, Daniela Tornaboni, and A Tognetti. Pulmonary and hepatic pathologies in the series of mummies of s. domenico maggiore at naples (xvi century). In Proceedings of the VII European Meeting of the Paleopathology Association (Lyon, September 1988), volume 1, pages 89-92. Marino Solfanelli Editore, Journal of Paleopathology, Monographic Publications, 1989.

14 Lawrence M Knab, Anne-Marie Boller, and David M Mahvi. Cholecystitis. Surgical Clinics, 94(2):455-470, 2014.

15 Niels Gerard Venneman and Karel Johannes van Erpecum. Pathogenesis of gallstones. Gastroenterology Clinics, 39(2):171-183, 2010.

16 Heewook Lee, Ellen Popodi, Haixu Tang, and Patricia L Foster. Rate and molecular spectrum of spontaneous mutations in the bacterium escherichia coli as determined by whole-genome sequencing. Proceedings of the National Academy of Sciences, 109(41):E2774-E2783, 2012.

17 Zhemin Zhou, Nabil-Fareed Alikhan, Khaled Mohamed, Yulei Fan, Mark Achtman, Derek Brown, Marie Chattaway, Tim Dallman, Richard Delahay, Christian Kornschober, et al. The enterobase user's guide, with case studies on Salmonella transmissions, Yersinia pestis phylogeny, and Escherichia core genomic diversity. Genome research, 30(1):138-152, 2020. 
18 Marie Touchon, Amandine Perrin, Jorge Andre Moura De Sousa, Belinda Vangchhia, Samantha Burn, Claire L O'Brien, Erick Denamur, David Gordon, and Eduardo PC Rocha. Phylogenetic background and habitat drive the genetic diversification of Escherichia coli. PLoS genetics, 16(6):e1008866, 2020.

19 Alistair B Russell, S Brook Peterson, and Joseph D Mougous. Type vi secretion system effectors: poisons with a purpose. Nature reviews microbiology, 12(2):137-148, 2014.

20 Bo Liu, Dandan Zheng, Qi Jin, Lihong Chen, and Jian Yang. Vfdb 2019: a comparative pathogenomic platform with an interactive web interface. Nucleic acids research, 47(D1):D687-D692, 2019.

21 Rosario Morales-Espinosa, Rigoberto Hernandez-Castro, Gabriela Delgado, Jose Luis Mendez, Armando Navarro, Angel Manjarrez, and Alejandro Cravioto. Upec strain characterization isolated from mexican patients with recurrent urinary infections. The Journal of Infection in Developing Countries, 10(0404):317-328, 2016. ISSN 1972-2680. doi: 10.3855/jidc.6652.

22 Mohammad Mehdi Aslani, Mohammad Yousef Alikhani, Ali Zavari, Rasol Yousefi, and Ali Reza Zamani. Characterization of enteroaggregative Escherichia coli (eaec) clinical isolates and their antibiotic resistance pattern. International Journal of Infectious Diseases, 15(2):e136-e139, 2011.

23 James R Johnson, Olivier Clermont, Megan Menard, Michael A Kuskowski, Bertrand Picard, and Erick Denamur. Experimental mouse lethality of Escherichia coli isolates, in relation to accessory traits, phylogenetic group, and ecological source. The Journal of infectious diseases, 194(8):1141-1150, 2006.

24 James R Johnson, Brian D Johnston, Stephen Porter, Paul Thuras, Maliha Aziz, and Lance B Price. Accessory traits and phylogenetic background predict Escherichia coli extraintestinal virulence better than does ecological source. The Journal of infectious diseases, 219(1):121-132, 2019.

25 Marco Galardini, Olivier Clermont, Alexandra Baron, Bede Busby, Sara Dion, Sören Schubert, Pedro Beltrao, and Erick Denamur. Major role of iron uptake systems in the intrinsic extra-intestinal virulence of the genus Escherichia revealed by a genome-wide association study. PLoS genetics, 16(10):e1009065, 2020.

26 Robert J Goldstone and David GE Smith. A population genomics approach to exploiting the accessory 'resistome' of Escherichia coli. Microbial genomics, 3(4), 2017.

27 Brian P Alcock, Amogelang R Raphenya, Tammy TY Lau, Kara K Tsang, Mégane Bouchard, Arman Edalatmand, William Huynh, Anna-Lisa V Nguyen, Annie A Cheng, Sihan Liu, et al. Card 2020: antibiotic resistome surveillance with the comprehensive antibiotic resistance database. Nucleic acids research, 48(D1):D517-D525, 2020 .

28 Alison M Devault, Tatum D Mortimer, Andrew Kitchen, Henrike Kiesewetter, Jacob M Enk, G Brian Golding, John Southon, Melanie Kuch, Ana T Duggan, William Aylward, et al. A molecular portrait of maternal sepsis from byzantine troy. Elife, 6:e20983, 2017.

29 Karl Friehs. Plasmid copy number and plasmid stability. New trends and developments in biochemical engineering, pages 47-82, 2004.

30 Vincent Daubin, Emmanuelle Lerat, and Guy Perrière. The source of laterally transferred genes in bacterial genomes. Genome biology, 4(9):1-12, 2003.

31 Roy R. Chaudhuri and Ian R. Henderson. The evolution of the Escherichia coli phylogeny. Infection, Genetics and Evolution, 12(2):214-226, 2012. ISSN 15671348. doi: 10.1016/j.meegid.2012.01.005.

32 M Desroches, G Royer, D Roche, M Mercier-Darty, D Vallenet, C Médigue, K Bastard, C Rodriguez, O Clermont, E Denamur, et al. The odyssey of the ancestral escherich strain through culture collections: an example of allopatric diversification. Msphere, 3(1), 2018.

33 M-C Wang, C-C Tseng, A-B Wu, J-J Huang, B-S Sheu, and J-J Wu. Different roles of host and bacterial factors in Escherichia coli extra-intestinal infections. Clinical microbiology and infection, 15(4):372-379, 2009.

34 Raoul Benveniste and Julian Davies. Mechanisms of antibiotic resistance in bacteria. Annual review of biochemistry, 42(1):471-506, 1973. 
35 Laura JV Piddock. Multidrug-resistance efflux pumps? not just for resistance. Nature Reviews Microbiology, 4 (8):629-636, 2006.

36 ITZHAK Brook. Aerobic and anaerobic microbiology of biliary tract disease. Journal of clinical microbiology, 27(10):2373-2375, 1989.

37 Carsten Schwarz, Regis Debruyne, Melanie Kuch, Elizabeth McNally, Henry Schwarcz, Andrew D Aubrey, Jeffrey Bada, and Hendrik Poinar. New insights from old bones: Dna preservation and degradation in permafrost preserved mammoth remains. Nucleic acids research, 37(10):3215-3229, 2009.

38 Jesse Dabney, Michael Knapp, Isabelle Glocke, Marie-Theres Gansauge, Antje Weihmann, Birgit Nickel, Cristina Valdiosera, Nuria García, Svante Pääbo, Juan-Luis Arsuaga, et al. Complete mitochondrial genome sequence of a middle pleistocene cave bear reconstructed from ultrashort dna fragments. Proceedings of the National Academy of Sciences, page 201314445, 2013.

39 Isabelle Glocke and Matthias Meyer. Extending the spectrum of dna sequences retrieved from ancient bones and teeth. Genome research, 2017.

40 Mikkel Schubert, Stinus Lindgreen, and Ludovic Orlando. Adapterremoval v2: rapid adapter trimming, identification, and read merging. BMC research notes, 9(1):88, 2016.

41 Gabriel Renaud, Udo Stenzel, and Janet Kelso. leehom: adaptor trimming and merging for illumina sequencing reads. Nucleic acids research, 42(18):e141-e141, 2014.

42 Derrick E Wood, Jennifer Lu, and Ben Langmead. Improved metagenomic analysis with kraken 2. Genome biology, 20(1):257, 2019.

43 Robert Schmieder and Robert Edwards. Quality control and preprocessing of metagenomic datasets. Bioinformatics, 27(6):863-864, 2011.

44 Valerie A Schneider, Tina Graves-Lindsay, Kerstin Howe, Nathan Bouk, Hsiu-Chuan Chen, Paul A Kitts, Terence D Murphy, Kim D Pruitt, Françoise Thibaud-Nissen, Derek Albracht, et al. Evaluation of grch38 and de novo haploid genome assemblies demonstrates the enduring quality of the reference assembly. Genome research, 27(5):849-864, 2017.

45 Heng Li and Richard Durbin. Fast and accurate short read alignment with burrows-wheeler transform. bioinformatics, 25(14):1754-1760, 2009.

46 Ana T Duggan, Maria F Perdomo, Dario Piombino-Mascali, Stephanie Marciniak, Debi Poinar, Matthew V Emery, Jan P Buchmann, Sebastian Duchêne, Rimantas Jankauskas, Margaret Humphreys, et al. 17th century variola virus reveals the recent history of smallpox. Current Biology, 26(24):3407-3412, 2016.

47 Alice R Wattam, James J Davis, Rida Assaf, Sébastien Boisvert, Thomas Brettin, Christopher Bun, Neal Conrad, Emily M Dietrich, Terry Disz, Joseph L Gabbard, et al. Improvements to patric, the all-bacterial bioinformatics database and analysis resource center. Nucleic acids research, 45(D1):D535-D542, 2017.

48 Torsten Seemann. Prokka: rapid prokaryotic genome annotation. Bioinformatics, 30(14):2068-2069, 2014.

49 Andrew J Page, Carla A Cummins, Martin Hunt, Vanessa K Wong, Sandra Reuter, Matthew TG Holden, Maria Fookes, Daniel Falush, Jacqueline A Keane, and Julian Parkhill. Roary: rapid large-scale prokaryote pan genome analysis. Bioinformatics, 31(22):3691-3693, 2015.

50 Hákon Jónsson, Aurélien Ginolhac, Mikkel Schubert, Philip LF Johnson, and Ludovic Orlando. mapdamage2. 0: fast approximate bayesian estimates of ancient dna damage parameters. Bioinformatics, 29(13):1682-1684, 2013.

51 Andaine Seguin-Orlando, Caroline Costedoat, Clio Der Sarkissian, Stéfan Tzortzis, Célia Kamel, Norbert Telmon, Love Dalén, Catherine Thèves, Michel Signoli, and Ludovic Orlando. No particular genomic features underpin the dramatic economic consequences of 17th century plague epidemics in italy. Iscience, 24(4):102383, 2021. 
52 Sergey Nurk, Anton Bankevich, Dmitry Antipov, Alexey Gurevich, Anton Korobeynikov, Alla Lapidus, Andrey Prjibelsky, Alexey Pyshkin, Alexander Sirotkin, Yakov Sirotkin, et al. Assembling genomes and minimetagenomes from highly chimeric reads. In Annual International Conference on Research in Computational Molecular Biology, pages 158-170. Springer, 2013.

53 James Robertson and John HE Nash. Mob-suite: software tools for clustering, reconstruction and typing of plasmids from draft assemblies. Microbial genomics, 4(8), 2018.

54 Olivier Clermont, Julia K Christenson, Erick Denamur, and David M Gordon. The clermont Escherichia coli phylo-typing method revisited: improvement of specificity and detection of new phylo-groups. Environmental microbiology reports, 5(1):58-65, 2013.

55 Astrid von Mentzer, Thomas R Connor, Lothar H Wieler, Torsten Semmler, Atsushi Iguchi, Nicholas R Thomson, David A Rasko, Enrique Joffre, Jukka Corander, Derek Pickard, et al. Identification of enterotoxigenic Escherichia coli (etec) clades with long-term global distribution. Nature genetics, 46(12):1321, 2014.

56 Thu-Hien To, Matthieu Jung, Samantha Lycett, and Olivier Gascuel. Fast dating using least-squares criteria and algorithms. Systematic Biology, 65(1):82-97, 2016. ISSN 1063-5157. doi: 10.1093/sysbio/syv068.

57 Sebastian Duchene, David A Duchene, Jemma L Geoghegan, Zoe A Dyson, Jane Hawkey, and Kathryn E Holt. Inferring demographic parameters in bacterial genomic data using bayesian and hybrid phylogenetic methods. BMC evolutionary biology, 18(1):1-11, 2018.

58 Michelle M Riehle, Albert F Bennett, and Anthony D Long. Genetic architecture of thermal adaptation in escherichia coli. Proceedings of the National Academy of Sciences, 98(2):525-530, 2001.

59 Damian Szklarczyk, Annika L Gable, David Lyon, Alexander Junge, Stefan Wyder, Jaime Huerta-Cepas, Milan Simonovic, Nadezhda T Doncheva, John H Morris, Peer Bork, et al. String v11: protein-protein association networks with increased coverage, supporting functional discovery in genome-wide experimental datasets. Nucleic acids research, 47(D1):D607-D613, 2019.

60 V de Lastours, C Laouénan, G Royer, E Carbonnelle, R Lepeule, M Esposito-Farèse, O Clermont, X Duval, B Fantin, F Mentré, et al. Mortality in Escherichia coli bloodstream infections: antibiotic resistance still does not make it. Journal of Antimicrobial Chemotherapy, 75(8):2334-2343, 2020.

61 Marie Touchon, Claire Hoede, Olivier Tenaillon, Valérie Barbe, Simon Baeriswyl, Philippe Bidet, Edouard Bingen, Stéphane Bonacorsi, Christiane Bouchier, Odile Bouvet, et al. Organised genome dynamics in the Escherichia coli species results in highly diverse adaptive paths. PLoS genet, 5(1):e1000344, 2009.

\section{Figures}

Figure 1: Characterization of both the physical sample and the authentication of the sequenced data. A) Liver and gallbladder of Giovani d'Avalos. The gallstones can be seen in the red rectangle. Note its dark brown colouring. B) Gallbladder with thickened wall (a) and Rokitansky-Aschoff sinuses (b) (Hematoxylin-eosin, 3X and 250X). C) Damage plots of the $5^{\prime}$ and $3^{\prime}$ ends of mapped reads for E. coli. Colours refer to the digest, not damage type. Mapdamage 2.0 $0^{50}$ was used to calculate the damage rates. D) Fragment length distribution of deduplicated mapped reads from E.coli. A $\log _{10}$ scale is used to emphasize the differences between the digests. A minimum length of $30 \mathrm{bp}$ was required for a read to be kept.

\section{Additional Files}

\section{Additional file $1-E$. coli strains}

List of E. coli strains used to create the pan-genomes and phylogenies 
Figure 2: Read coverage metrics for the E. coli pan-genome in comparison to other genomes. A) Distribution of mean gene coverages with a $\mathrm{CV} \leq 1$ for the ancient genome. The dashed line indicates the detection threshold at $10 \times$. The black rectangle on the right indicates the area where genes with a high copy number (as defined by $\bar{x}+2 * s$ ) B) Coverage plot for FSIS11816402. A window of $1 \%$ was used for illustration purposes. C) Coverage plot for CP019906.1. A window of $0.1 \%$ was used for illustration purposes. The first track indicates the coverage with the red line illustrating the overall mean. The second track indicates the number SNPs over the same window while the third is the GC content. D) Gene coverage over the T6SS for K. aerogenes using a 100bp window. Gene names are included when available.

Figure 3: Maximum likelihood SNP phylogeny of E. coli. A) The global phylogeny with bootstrap values and phylogenetic groups with E. coli EC42405 as the out-group. B) Phylogeny of the reduced subgroup A0 as defined by the Clermont genotype $(+\ldots-)^{54}$. Tip points represent the sequence type of the strain. IAI1 was the out-group of the phylogeny. C) Phylogeny of the ST4995 strains. Red rectangles represent the $95 \%$ confidence interval for the topology; labels indicate the median date of divergence. The evolutionary rate for the phylogeny was $2.555 \times 10^{-6}\left[1.567 \times 10^{-6}, 3.992 \times 10^{-6}\right]$ subs/site/year. CFSAN051544 was the out-group.

Figure 4: Principle Coordinate Analyses (PCoA) of gene Presence/Absence data. The accessory genome $(\mathbf{A}, \mathbf{C})$ and identified virulence genes $(\mathbf{B}, \mathbf{D})$ were clustered using a binary distance. Phylogroups were identified using Clermont Typing while pathovars were determined using available metadata from the Patric database. The ancient genome is indicated by the black arrow. In both binary distances were calculated prior to creating the PCoA.

\section{Additional file 2 - Plasmid Gene Coverage and comparison of the ancient strain to FSIS11816402}

Identified genes in the plasmids 
A

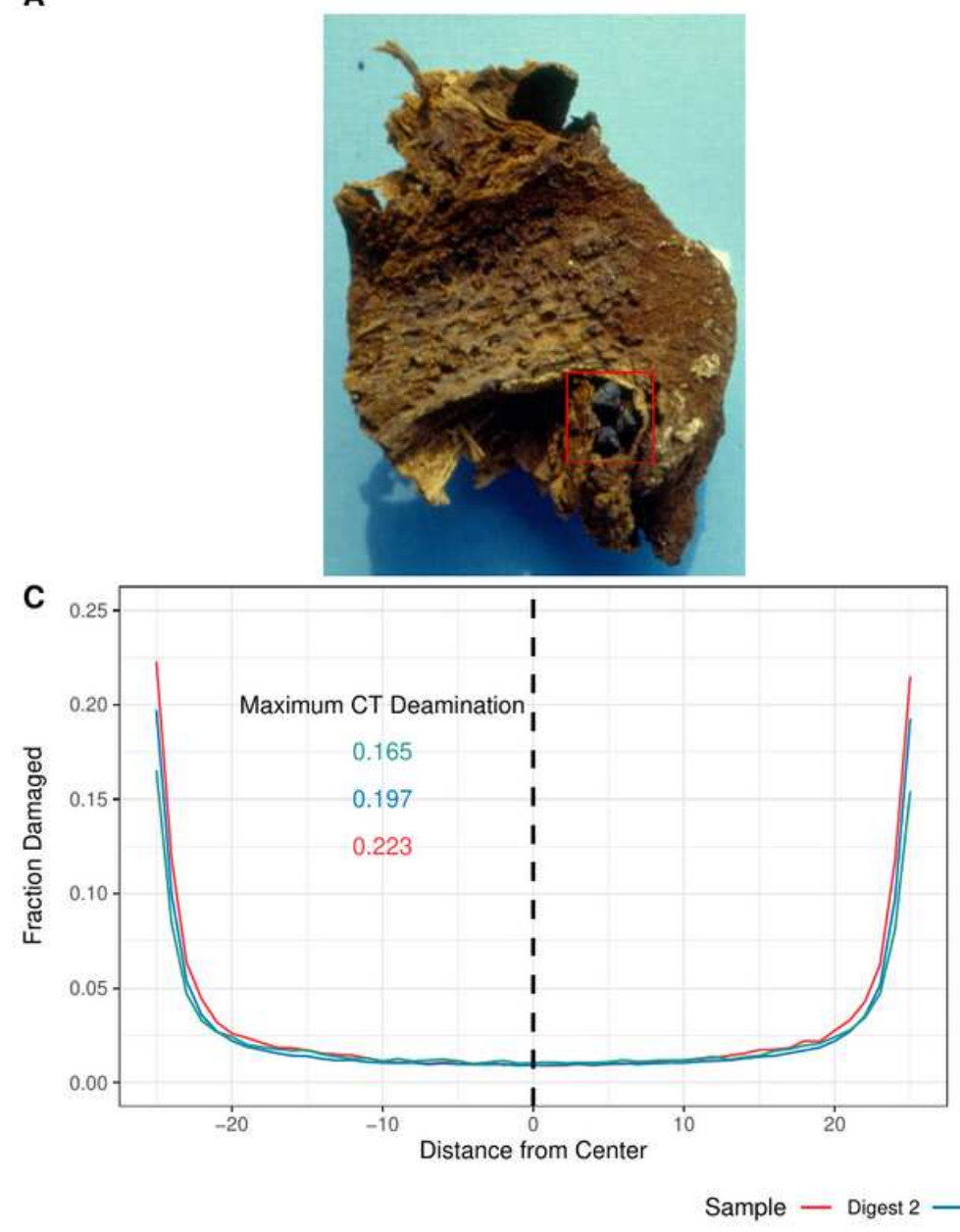

B

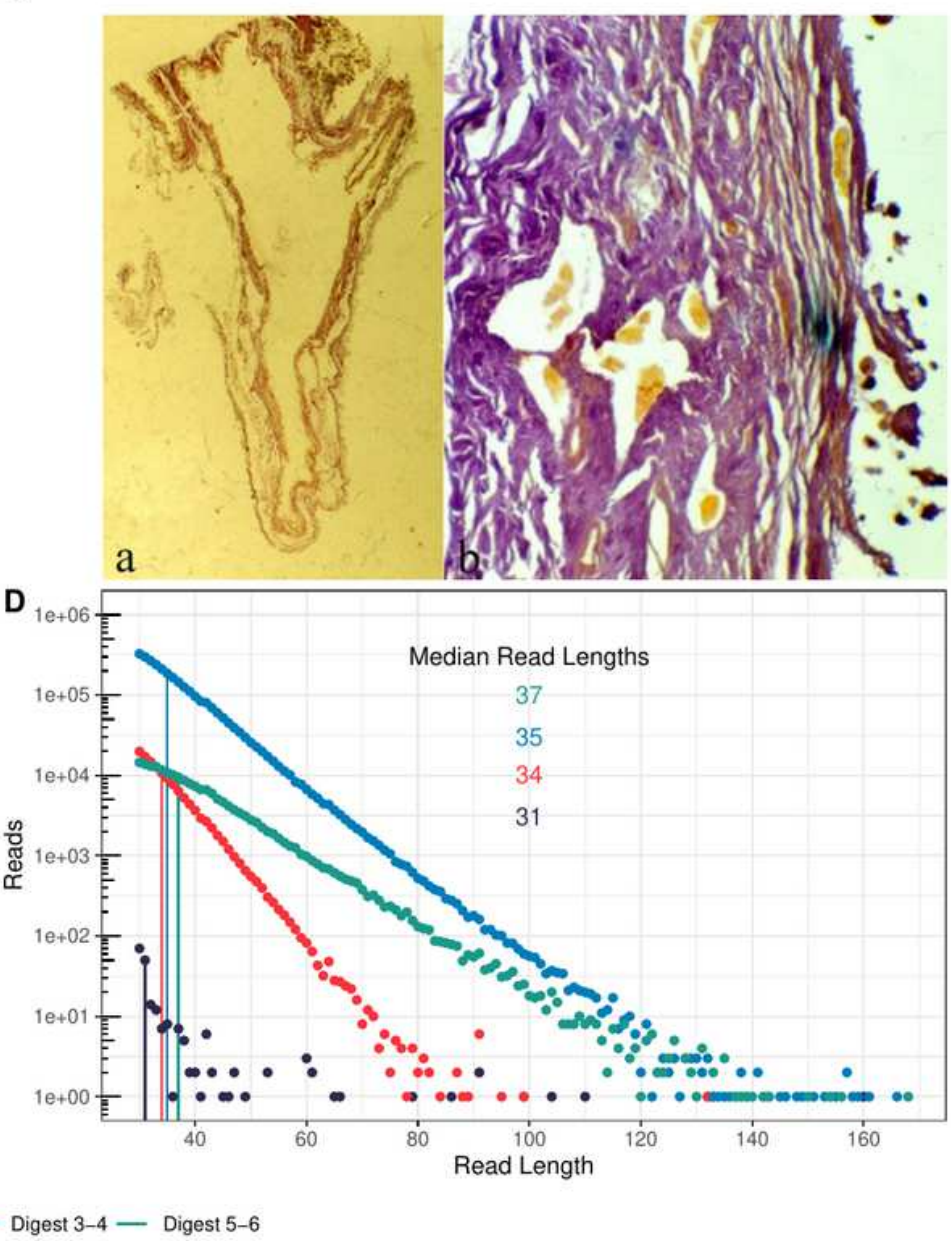

Figure 1

Characterization of both the physical sample and the authentication of the sequenced data. A) Liver and gallbladder of Giovani d'Avalos. The gallstones can be seen in the red rectangle. Note its dark brown colouring. B) Gallbladder with thickened wall (a) and Rokitansky-Ascho sinuses (b) (Hematoxylin-eosin, $3 X$ and 250X). C) Damage plots of the 50 and 30 ends of mapped reads for E. coli. Colours refer to the digest, not damage type. Mapdamage 2.050 was used to calculate the damage rates. D) Fragment length distribution of deduplicated mapped reads from E. coli. A log10 scale is used to emphasize the differences between the digests. A minimum length of $30 \mathrm{bp}$ was required for a read to be kept. 

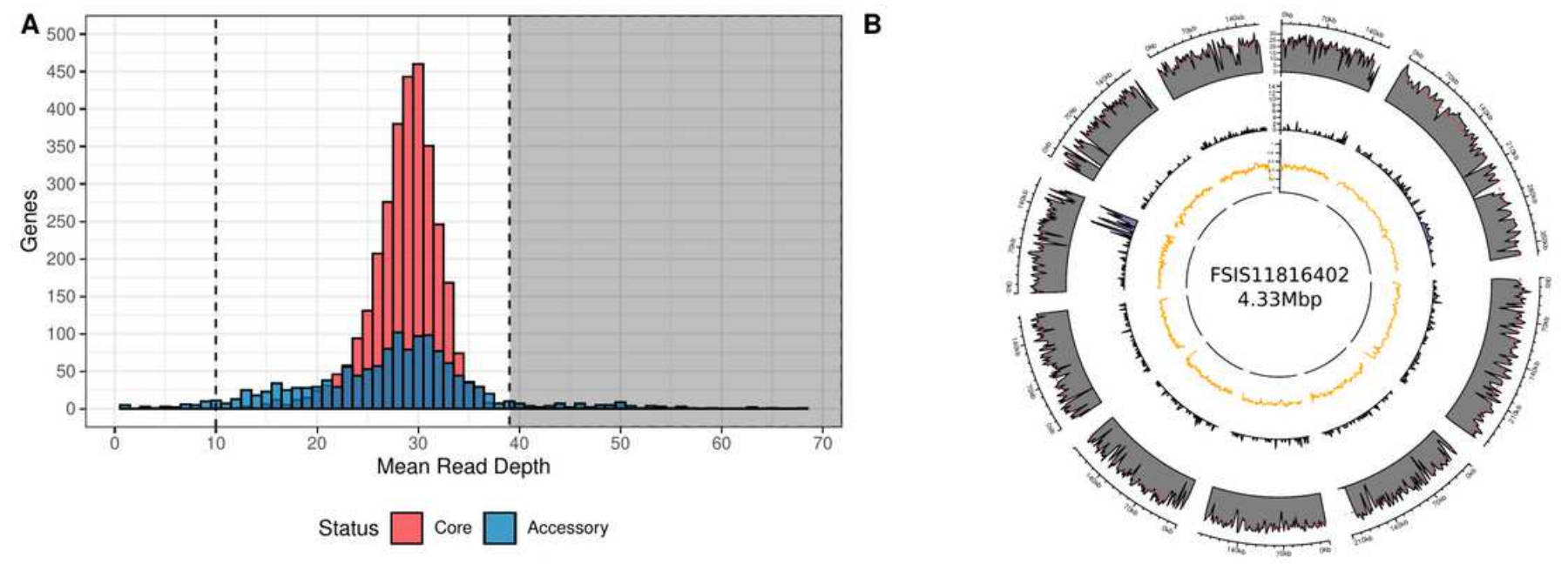

C
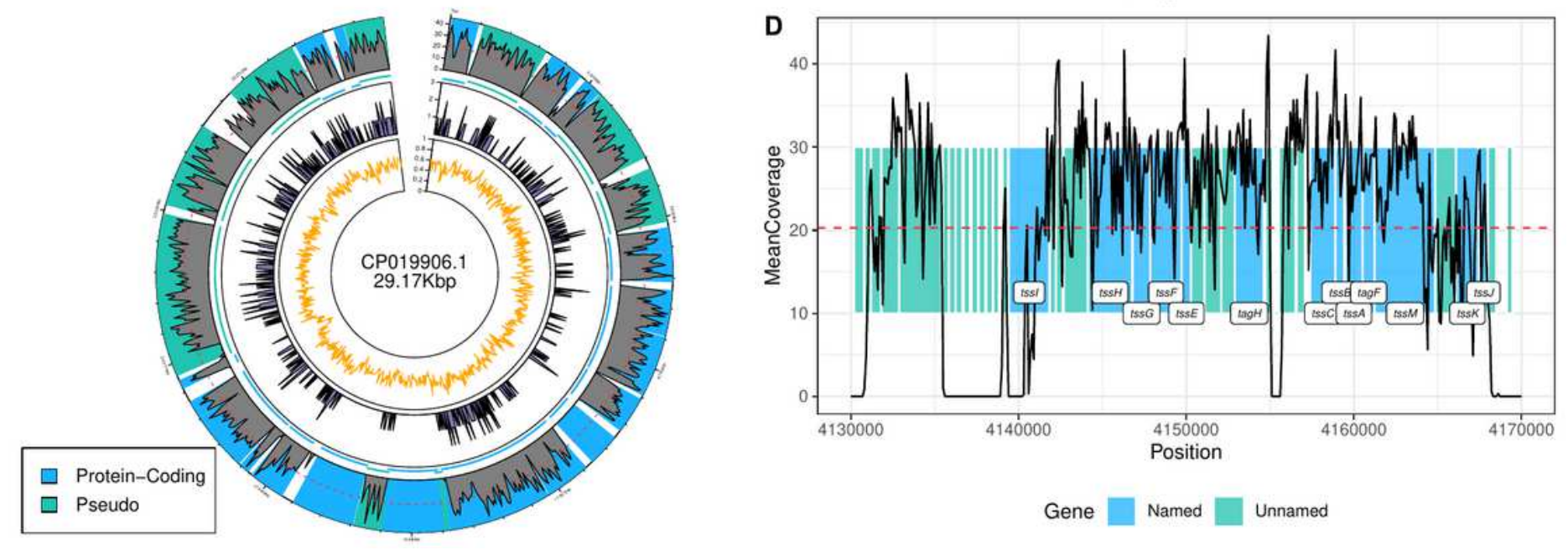

Figure 2

Read coverage metrics for the E. coli pan-genome in comparison to other genomes. A) Distribution of mean gene coverages with a $\mathrm{CV} \leq 1$ for the ancient genome. The dashed line indicates the detection threshold at 10x. The black rectangle on the right indicates the area where genes with a high copy number (as defined by $x \mathbb{x}+2 * \mathrm{~s}$ ) B) Coverage plot for FSIS11816402. A window of $1 \%$ was used for illustration purposes. C) Coverage plot for CP019906.1. A window of $0.1 \%$ was used for illustration purposes. The rst track indicates the coverage with the red line illustrating the overall mean. The second track indicates the number SNPs over the same window while the third is the GC content. D) Gene coverage over the T6SS for K. aerogenes using a 100bp window. Gene names are included when available. 
A

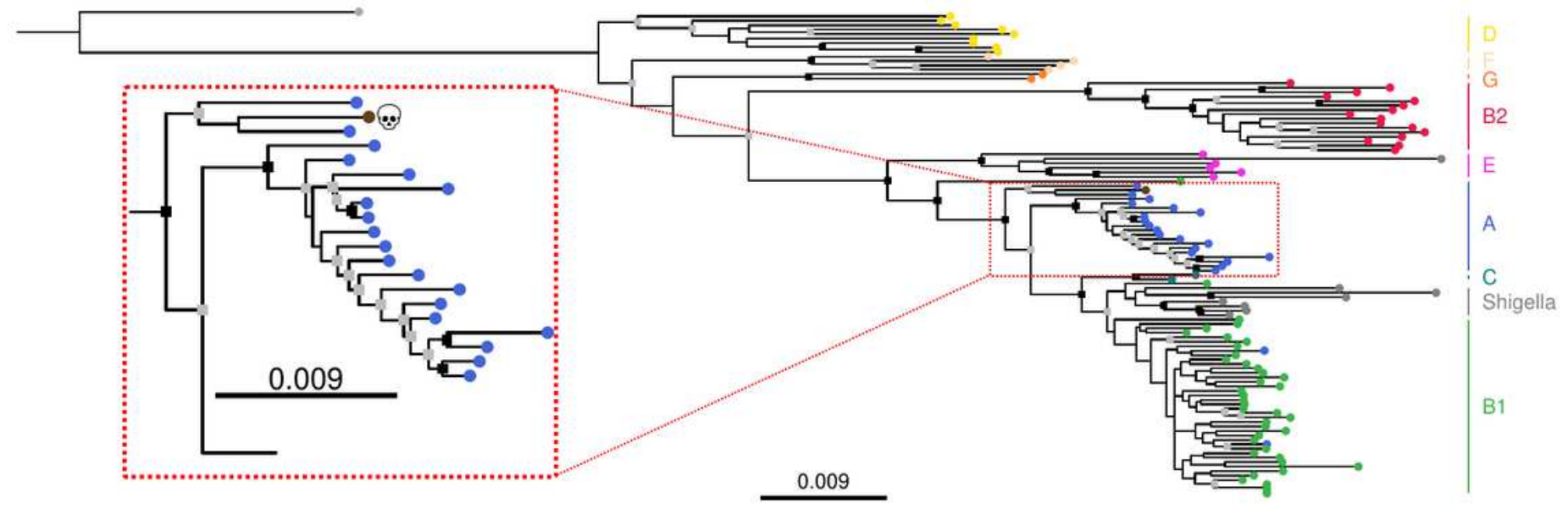

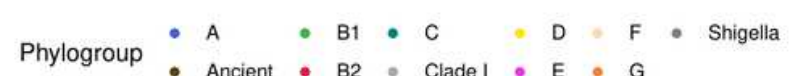

B

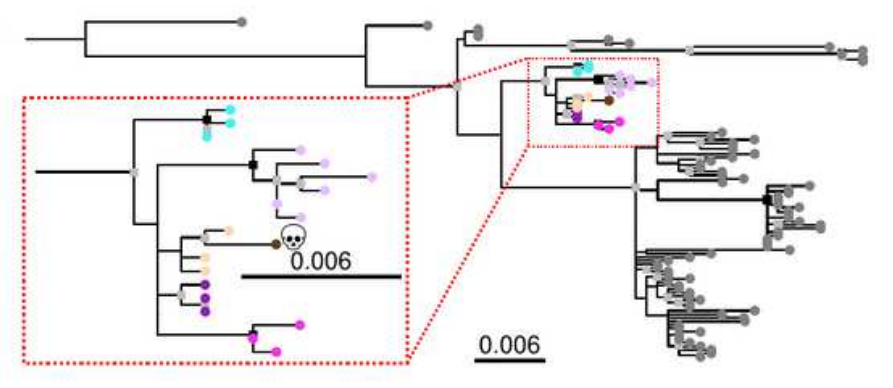

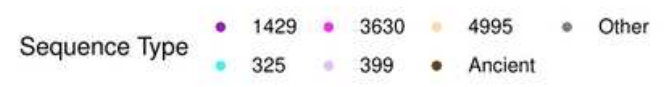

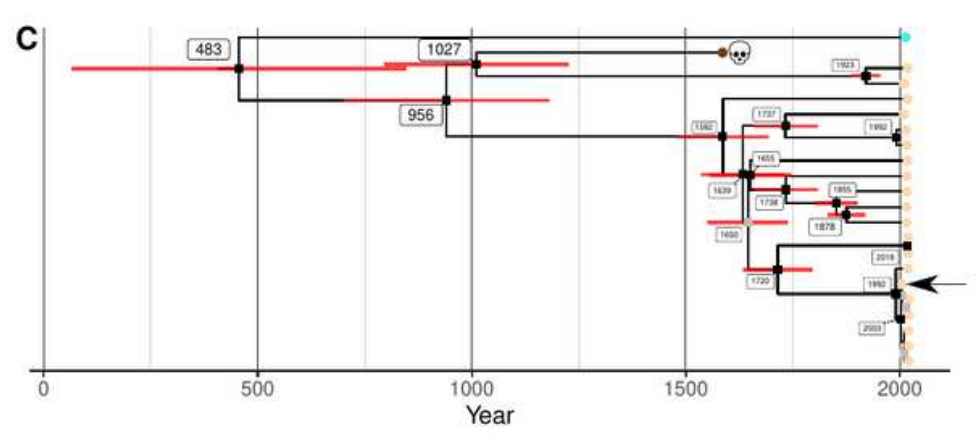

Sequence Type * $325-4995$ - Ancient

\section{Figure 3}

Maximum likelihood SNP phylogeny of E. coli. A) The global phylogeny with bootstrap values and phylogenetic groups with E. coli EC42405 as the out-group. B) Phylogeny of the reduced subgroup A0 as dened by the Clermont genotype (+-- - ) 54. Tip points represent the sequence type of the strain. IAl1 was the out-group of the phylogeny. C) Phylogeny of the ST4995 strains. Red rectangles represent the $95 \%$ condence interval for the topology; labels indicate the median date of divergence. The evolutionary rate for the phylogeny was $2.555 \times 10-6[1.567 \times 10-6,3.992 \times 10-6]$ subs/site/year. CFSAN051544 was the out-group. 

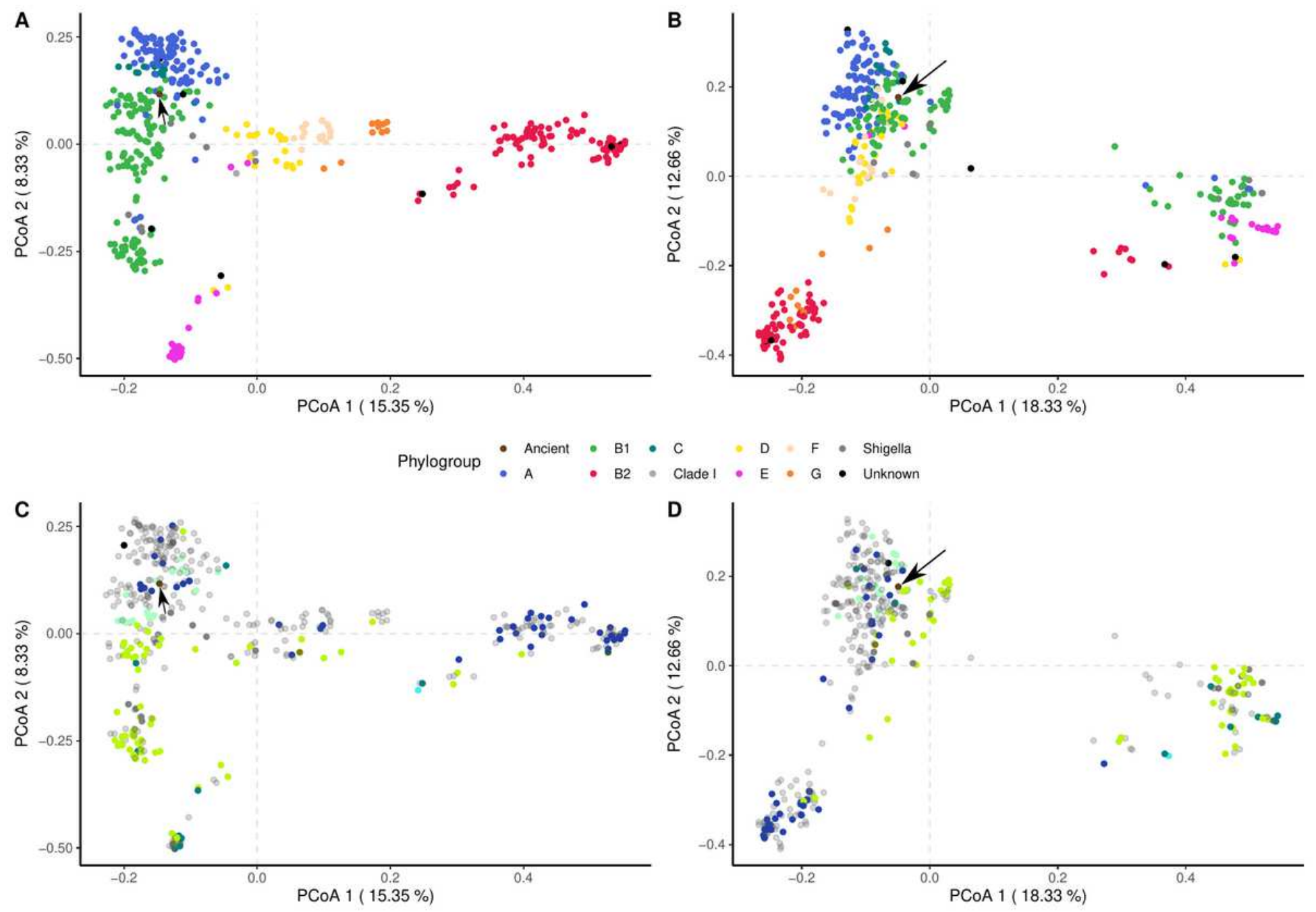

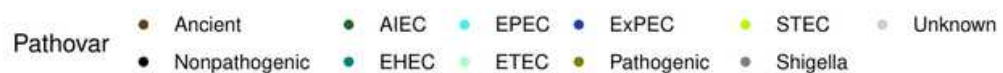

Figure 4

Principle Coordinate Analyses (PCOA) of gene Presence/Absence data. The accessory genome $(A, C)$ and identied virulence genes $(B, D)$ were clustered using a binary distance. Phylogroups were identied using Clermont Typing while pathovars were determined using available metadata from the Patric database. The ancient genome is indicated by the black arrow. In both binary distances were calculated prior to creating the PCOA.

\section{Supplementary Files}

This is a list of supplementary files associated with this preprint. Click to download.

- Appendix.pdf

- SupFig1.jpg

- SupFig101.tif

- SupFig111.tif 
- SupFig121.tif

- SupFig131.tif

- SupFig151.tif

- SupFig161.tif

- SupFig21.tif

- SupFig31.tif

- SupFig41.tif

- SupFig51.tif

- SupFig61.tif

- SupFig71.tif

- SupFig81.tif

- SupFig91.tif

- SuppTable1.xlsx

- SuppTable2.xIsx 